\title{
Updated folate data in the Dutch Food Composition Database and implications for intake estimates
}

\author{
Susanne Westenbrink*, Martine Jansen-van der Vliet and \\ Caroline van Rossum
}

National Institute for Public Health and the Environment (RIVM), Bilthoven, The Netherlands

Abstract

Background and objective: Nutrient values are influenced by the analytical method used. Food folate measured by high performance liquid chromatography (HPLC) or by microbiological assay (MA) yield different results, with in general higher results from MA than from HPLC. This leads to the question of how to deal with different analytical methods in compiling standardised and internationally comparable food composition databases? A recent inventory on folate in European food composition databases indicated that currently MA is more widely used than HPCL. Since older Dutch values are produced by HPLC and newer values by MA, analytical methods and procedures for compiling folate data in the Dutch Food Composition Database (NEVO) were reconsidered and folate values were updated. This article describes the impact of this revision of folate values in the NEVO database as well as the expected impact on the folate intake assessment in the Dutch National Food Consumption Survey (DNFCS).

Design: The folate values were revised by replacing HPLC with MA values from recent Dutch analyses. Previously MA folate values taken from foreign food composition tables had been recalculated to the HPLC level, assuming a $27 \%$ lower value from HPLC analyses. These recalculated values were replaced by the original MA values. Dutch HPLC and MA values were compared to each other. Folate intake was assessed for a subgroup within the DNFCS to estimate the impact of the update.

Results: In the updated NEVO database nearly all folate values were produced by MA or derived from MA values which resulted in an average increase of $24 \%$. The median habitual folate intake in young children was increased by $11-15 \%$ using the updated folate values.

Conclusion: The current approach for folate in NEVO resulted in more transparency in data production and documentation and higher comparability among European databases. Results of food consumption surveys are expected to show higher folate intakes when using the updated values.

Keywords: analytical method; food composition; NEVO; HPLC; microbiological assay; food intake; food consumption survey; children

$\mathrm{T}$ otal folate intake is a combination of intake from food folate plus folic acid from fortified foods and food supplements. The importance of folate's/folic acid's role in public health is well known. Total folate intake is, for example, associated with reducing the risk of neural tube defects, macrocytic anemia, cancer and neurological disorders due to undiagnosed vitamin $B_{12}$ deficiency (1). To measure total folate intake, reliable and accurate food composition data are needed. To monitor folate intake in the Dutch National Food Consumption Survey (DNFCS), folate was added to the Dutch Food Composition Database (NEVO) in the year 2000.
High performance liquid chromatography (HPLC) was at that time selected as the preferred analytical method for folate for the NEVO database. It was considered to be the most accurate method for folate determination and was assumed to be the generally accepted method for the future. A practical consideration was that in 2000 for 125 Dutch foods up-to-date HPLC values were available, that were sampled taking into account food consumption data and market shares $(2,3)$.

Foreign food composition tables, in particular the UK McCance \& Widdowson's 'The Composition of Foods', were used to fill in missing values. Folate values 
in these tables were analysed using microbiological assay (MA). As the HPLC method yields lower folate values compared with MA, the values obtained from the UK tables were reduced by $27 \%$ to make them comparable with the HPLC folate values in the NEVO database (4). Any residual missing values were copied from similar foods within the database or calculated from recipes.

A recent inventory on folate in international food composition databases (Bouckaert $\mathrm{K}$, personal communication) showed that MA is the most widely used analytical method generally used to produce folate values for food composition databases in Europe and beyond. Moreover, since 2000 all folate analyses in the Netherlands are carried out by MA and not by HPCL. Another important finding from this inventory was the limited transparency in data production and documentation of folate values in NEVO, specifically with respect to MA values that were adjusted to HPLC values. An important objection was that this approach, although based on the same data sources, resulted in different values across Europe.

The relevance of producing standardised and harmonised food composition tables was shown by Deharveng (5) and Slimani $(6,7)$ who compared food composition databases of partner countries in the EPIC study and subsequently worked on standardising food composition datasets for 10 European countries. In 1999, the conclusion was that reliable folate intake could not be estimated using these 10 European tables (5). The EuroFIR project (2005-2010), which aimed at harmonising and standardising food composition data in Europe, has worked towards creating comparable databases for future pan-European food consumption research (8). The task to achieve greater standardisation and a greater comparability between databases is important. Therefore in 2009 , the process of collecting and recompiling folate data in NEVO was considered. It was decided that from 2010 onwards MA will be the standard analytical method for folate. HPLC values will be replaced by MA values if available and the adjusted values from the UK (and other) tables will be replaced with their original MA values.

This article describes the impact of the update on the folate values in NEVO as well as the expected impact on the folate intake assessment within the DNFCS.

\section{Methods}

\section{Update of folate values in NEVO}

The folate values as published in the NEVO 2006 table were revised by replacing HPLC with MA values from recent Dutch analyses or with the original MA values from foreign tables.

\section{New Dutch analytical folate values}

Recently folate was analysed in 188 non-fortified food items. In September 2007, 37 types of bread were sampled and in 2008, from October to December, 149 foods from various food groups were sampled. All foods were purchased at supermarkets and in small retail shops, such as bakery, butcher and grocery shops. Foods were purchased in four regions [North, East, South and West, based on the classification of Statistics Netherlands (9)], in smaller and larger cities (less than and more than 60,000 inhabitants, respectively). The supermarkets and retailers were selected reflecting their market share (10). For each type of bread, 40 samples were bought and mixed into a composite sample. For the other food items, a composite sample was made based on the edible part of at most 16 samples taken. Industrial brand foods with a relatively constant food composition were sampled only at supermarkets, resulting in at most eight samples for these foods.

The food was prepared in accordance to standard household methods, and samples were frozen in liquid nitrogen and homogenised. Folate was analysed following the AOAC trienzyme MA (11).

For all these food items the previous folate values were replaced by the new MA values.

\section{Folate values from other data sources}

Original MA values from the latest (electronic) version of the UK food composition tables were used to replace the values that had been previously adjusted to the assumed $27 \%$ lower level of HPLC values (12).

For fresh meat a different approach was chosen. Meat cuts in the Netherlands are not the same as meat cuts in the United Kingdom which makes identification from foreign data sources difficult. In 2000 individual values were obtained (and adjusted) from the UK tables, whereas in 2010 average folate values were calculated from the UK table for raw and cooked meat from several species (pork, lamb, chicken, veal) and for several types of bacon. This could be done because variation in folate contents within each type of meat was small.

Missing values were filled in by recipe calculations or by copying values from similar foods. For the update the folate values were recalculated or changed according to the changes made to the folate values of the original food items. Furthermore, folic acid enriched foods were not updated.

\section{Impact on folate values in NEVO database}

To estimate the impact of the update, values published in the printed NEVO table 2006 were compared with values available in the most recent version of NEVO online 2010/2.0 (13, 14). In particular, absolute and relative differences for folate analysed with HPLC in the NEVO table 2006 and MA in the NEVO online 2010/2.0 dataset were calculated. 


\section{Impact on folate intake assessment in DNFCS}

To study the impact of changes in folate values, intake was estimated with data from the DNFCS-young children. The methodology of the DNFCS-young children is described in detail elsewhere $(15,16)$. For this comparison habitual folate intake was estimated using the folate values from NEVO table 2006 and the updated NEVO online 2010/2.0. This estimation of habitual intake is developed by Nusser (17). Intake of folate from dietary supplements was not taken into account for the comparison.

\section{Results}

\section{Impact on compiled folate values}

Characteristics of the NEVO before and after the update are shown in Table 1. In the dataset NEVO online 2010/ 2.0 the majority of the Dutch analytical folate values were produced by MA $(72 \%)$, in contrast to $13 \%$ in 2006 . The remaining $28 \%$ HPLC values are not yet replaced because these foods were not included in the recent analytical protocols. All adjusted folate values from the UK food composition table were replaced by the original MA values. The percentage of values that in 2010 were derived from Dutch HPLC values (by calculation or estimation) and that have not been replaced by MA based values was only $3 \%$. Table 2 shows a comparison between folate values for 43 Dutch food items published in the NEVO table 2006 and in NEVO online 2010/2.0, for which analytical values (HPLC in 2006 and MA in 2010) were available. Values were produced with separate samples in different analytical projects.

The majority of the 2010 MA values were higher than the 2006 HPLC values. The average percentage difference was $23.5 \%$ (MA higher than HPLC). However, the range was very large and varied between differences of $-100 \%$ and $+233 \%$ for individual food items.

The average percentage difference in folate content calculated over all foods in the database, regardless of the data source, was similar to that based on analytical values alone $(24 \%$ higher in 2010 ; foods with missing values in 2006 or 2010 were excluded). Again the range in differences was large, due to large differences for some individual food items (data not shown).

\section{Impact on folate intake}

Median habitual folate intake of young Dutch children increased by $11-15 \%$ with the updated folate values from the NEVO online 2010/2.0 dataset (Table 3). The increase was higher in the 4-6-year-old $(15 \%)$ than in the $2-3$ year-old children (12 and 11\% for boys and girls, respectively). As folate equivalents are used to express the adequate intakes, intake of folate equivalents was calculated as well (folate equivalents $=$ folate $+(1.7 *$ folic acid from food $)+(2.0 *$ folic acid from supplements $)$ ). The update of folate values in our database only included folate values for non-fortified foods and therefore the intake of synthetic folic acid did not differ between both datasets. The intake of folate equivalents did increase, although less pronounced than for folate.

The median habitual intake of folate equivalents varied between 111 and $134 \mu \mathrm{g}$ per day calculated with the 2010 dataset. Compared with the adequate intake (AI) for these age groups (2-3 year old: $85 \mu \mathrm{g}$ and 4-6 year old: $150 \mu \mathrm{g}$ ) the percentage of inadequate folate intakes from food was assumed to be low for the 2-3-year-olds. For 4-6-year-olds the median habitual intake was lower than the adequate intake. For this group no conclusion on the prevalence of inadequacy could be given, since estimates of nutrient inadequacy based on intakes below the AI would be overestimates of the true prevalence (18).

Food groups contributing most to folate intake of young children were cereal products, dairy products, fruits, vegetables and meat products. The average contribution from fruit and meat products was lower when calculated with the 2010 values, due to lower folate values

Table 1. Characteristics of NEVO database 2006 and 2010 for folate

\begin{tabular}{|c|c|c|c|c|c|c|}
\hline \multirow[b]{2}{*}{ Food items (excluding foods for clinical use) } & \multicolumn{3}{|c|}{$2006(n=1489)$} & \multicolumn{3}{|c|}{$2010(n=1604)$} \\
\hline & $\%$ & $\%$ & $\%$ & $\%$ & $\%$ & $\%$ \\
\hline Folate values available for all foods & 86 & & & 88 & & \\
\hline Dutch analytical folate values & & 16 & & & 20 & \\
\hline Dutch analytical HPLC values & & & 87 & & & 28 \\
\hline Dutch analytical MA values & & & 13 & & & 72 \\
\hline Folate values from other sources & & 84 & & & 80 & \\
\hline UK values converted to HPLC level ( $-27 \%)$ & & & 51 & & & 0 \\
\hline Original UK values & & & 0 & & & 49 \\
\hline Miscellaneous data sources ${ }^{\mathrm{a}}$ & & & 49 & & & 51 \\
\hline Missing folate values for all foods & 14 & & & 12 & & \\
\hline
\end{tabular}

${ }^{\text {a } F o r e i g n ~ f o o d ~ c o m p o s i t i o n ~ t a b l e s, ~ c o p i e d ~ f r o m ~ s i m i l a r ~ f o o d s, ~ c a l c u l a t e d ~ b y ~ r e c i p e, ~ e x p e r t ~ a s s u m p t i o n s . ~}$ 
Table 2. Differences in analytical folate values between NEVO 2006 and NEVO 2010

\begin{tabular}{|c|c|c|c|c|c|}
\hline \multirow[b]{2}{*}{ NEVO id } & \multirow[b]{2}{*}{ Food name } & \multirow{2}{*}{$\begin{array}{c}\text { NEVO online } 2010^{\mathrm{a}} \\
\mu \mathrm{g} / 100 \mathrm{~g}\end{array}$} & \multirow{2}{*}{$\begin{array}{l}\text { NEVO table } 2006^{\mathrm{b}, \mathrm{c}} \\
\mu \mathrm{g} / \mathrm{l} 00 \mathrm{~g}\end{array}$} & \multicolumn{2}{|c|}{ Difference (2006 is reference) } \\
\hline & & & & $\mu g / / 00 \mathrm{~g}$ & $\%$ \\
\hline 204 & Peanuts unsalted & 57 & 17 & 40 & 235 \\
\hline 63 & Onions raw & 24 & 10 & 14 & 140 \\
\hline 64 & Onions boiled & 21 & 9 & 12 & 133 \\
\hline 241 & Bread white w milk & 37 & 17 & 20 & 118 \\
\hline 60 & Tomatoes raw & 16 & 8 & 8 & 100 \\
\hline 171 & Orange & 33 & 18 & 15 & 83 \\
\hline 244 & Bread raisin & 29 & 16 & 13 & 81 \\
\hline 233 & Bread currant & 29 & 16 & 13 & 81 \\
\hline 390 & Beer pilsner & 9 & 5 & 4 & 80 \\
\hline 486 & Flan with fruit filling & 9 & 5 & 4 & 80 \\
\hline 165 & Mandarins & 23 & 13 & 10 & 77 \\
\hline 15 & Cauliflower boiled & 95 & 55 & 40 & 73 \\
\hline 14 & Cauliflower raw & 67 & 44 & 23 & 52 \\
\hline 52 & Spinach boiled & 125 & 83 & 42 & 51 \\
\hline 243 & Rye bread light & 27 & 18 & 9 & 50 \\
\hline 1056 & Kiwi fruit & 34 & 23 & 11 & 48 \\
\hline 951 & Beans French boiled & 51 & 36 & 15 & 42 \\
\hline 1014 & Rice brown boiled & 7 & 5 & 2 & 40 \\
\hline 68 & Chicory boiled & 25 & 19 & 6 & 32 \\
\hline 51 & Spinach raw & $|3|$ & 100 & 31 & 31 \\
\hline 242 & Rye bread dark & 30 & 23 & 7 & 30 \\
\hline 27 & Cucumber raw no peel & 6 & 5 & 1 & 20 \\
\hline 230 & Roll white soft & 45 & 39 & 6 & 15 \\
\hline 410 & Juice orange & 22 & 20 & 2 & 10 \\
\hline 55 & Brussels sprouts boiled & 95 & 87 & 8 & 9 \\
\hline 8 & Endive boiled & 45 & 42 & 3 & 7 \\
\hline 920 & Broccoli boiled & 69 & 65 & 4 & 6 \\
\hline 383 & Juice apple & 0 & 0 & 0 & 0 \\
\hline 236 & Bread brown wheat & 27 & 27 & 0 & 0 \\
\hline 248 & Bread white w water & 22 & 25 & -3 & -12 \\
\hline 240 & Dutch spiced honey cake & 7 & 8 & -1 & -13 \\
\hline 884 & Sweet pepper red raw & 41 & 55 & -14 & -25 \\
\hline 37 & Leek boiled & 41 & 58 & -17 & -29 \\
\hline 7 & Endive raw & 35 & 50 & -15 & -30 \\
\hline 46 & Lettuce raw & 28 & 43 & -15 & -35 \\
\hline 151 & Banana & 9 & 16 & -7 & -44 \\
\hline 67 & Chicory raw & 10 & 23 & -13 & -57 \\
\hline 642 & Pâté & 62 & 147 & -85 & -58 \\
\hline 1399 & Lettuce iceberg raw & 14 & 42 & -28 & -67 \\
\hline 640 & Liver sausage & 60 & 207 & -147 & -71 \\
\hline 658 & Rice white boiled & 5 & 21 & -16 & -76 \\
\hline 31 & Sweet pepper green raw & $\mathrm{II}$ & 55 & -44 & -80 \\
\hline 285 & Coffee creamer half fat liquid & 0 & 5 & -5 & -100 \\
\hline
\end{tabular}

${ }^{a}$ Analysed in $2007-2008$ by microbiological assay.

${ }^{\mathrm{b}}$ Analysed by Konings (3) using HPLC.

${ }^{\mathrm{c}}$ Analysed by Vahteristo (23) using HPLC. 
Table 3. Distribution of habitual intake of folate and folate equivalents ${ }^{\mathrm{a}}$ ( $\left.\mu \mathrm{g} / \mathrm{day}\right)$ in Dutch children aged 2-6 years calculated with NEVO table 2006 and NEVO online 2010/2.0

\begin{tabular}{|c|c|c|c|c|c|c|c|c|c|}
\hline & \multirow[b]{2}{*}{$n$} & \multicolumn{4}{|c|}{2006} & \multicolumn{4}{|c|}{2010} \\
\hline & & P5 & P50 & P95 & P5 & P50 & P95 & $\%$ increase at P50 & $\mathrm{Al}^{\mathrm{b}}$ \\
\hline \multicolumn{10}{|l|}{ 2-3-year-old boys } \\
\hline Folate & 327 & 71 & 103 & 149 & 76 & 115 & 172 & 12 & \\
\hline Folate equivalents & 327 & 75 & 106 & 152 & 80 & 118 & 166 & 11 & 85 \\
\hline \multicolumn{10}{|l|}{ 2-3-year-old girls } \\
\hline Folate & 313 & 70 & 97 & 130 & 76 & 108 & 146 & 11 & \\
\hline Folate equivalents & 313 & 68 & 102 & 150 & 76 & 111 & 157 & 9 & 85 \\
\hline \multicolumn{10}{|l|}{4 6-year-old boys } \\
\hline Folate & 327 & 74 & 111 & 162 & 85 & 128 & 185 & 15 & \\
\hline Folate equivalents & 327 & 77 & 117 & 173 & 89 & 134 & 195 & 15 & 150 \\
\hline \multicolumn{10}{|l|}{4 6-year-old girls } \\
\hline Folate & 312 & 64 & 99 & 147 & 74 & 114 & 164 & 15 & \\
\hline Folate equivalents & 312 & 66 & 106 & 161 & 77 & 120 & 178 & 13 & 150 \\
\hline
\end{tabular}

${ }^{\text {a }}$ Folate equivalents $=$ folate $+(I .7 *$ folic acid from food $)+(2.0 *$ folic acid from supplements $)$; supplements were not taken into account in these calculations.

${ }^{\mathrm{b}} \mathrm{Al}$ : adequate intake.

in some (frequently eaten) fruits and in liver-based cured meat products (data not shown).

\section{Discussion}

This study shows that changing from HPLC to MA as the method to produce folate values has an impact on folate values in the NEVO database and subsequently on the estimated intake in DNFCS.

When conclusions from the comparison of old and new folate data in the NEVO database are drawn, several aspects need to be considered, such as analytical methods, food identification and sampling protocols.

On average our findings among the 43 compared Dutch food items are in line with the difference between HPLC and MA values as suggested by Konings (3). However, in our study some of the differences between 2006 and 2010 values are much larger. Several explanations can be given for this. Although the foods analysed in both protocols are generally considered to be similar, sampling procedures were not. Important areas of variation are found such as in the choice of foods at a detailed level (varieties, brands), seasonal variation, changes in processing by manufacturers, household cooking before homogenisation and sample preparation in the laboratory.

Another important explanation could be the variability within and between analytical methods and laboratories. The data collected for NEVO are usually produced by scientific laboratories where food items were analysed for other purposes. Although all data are scrutinised for the analytical method used, there is a risk of inconsistency in methods and quality, that is, accuracy and precision. Interlaboratory studies, in which all steps prior to analyses are standardised, show large within and between laboratory variation when using MA or other methods (e.g. 19, 20). In addition, conclusions from these studies show that laboratories do not strictly follow the official AOAC method, but use modifications indicating that more standardisation among laboratories is needed.

We agree with the statement of Koontz (19) that food composition database compilers, who are not trained analytical chemists (need to) trust the laboratory to conduct good quality controlled analyses and to use methods with demonstrated validity. Better trained compilers and close cooperation with trained chemical analysts would be needed to produce food composition databases with accurate and precise folate data.

Besides these factors that influence folate values in foods, theoretically, also true trend differences could have occurred. Since mainly basic foods were analysed, like fresh vegetables, fruits, eggs, bread, fish etc, the impact of this is assumed to be limited and there is no indication that varieties with higher or lower folate values have been developed.

A major part of our folate update was the replacement of MA values that were adjusted to HPLC level in 2000 with the original MA values from the latest UK food composition tables. In 2000 MA values were reduced by $27 \%$ to yield HPLC equivalents, thus this reversal results on average in a similar increase in folate for the foods concerned. The $27 \%$ decrease that was applied to UK folate values, back in 2000, was however not calculated by analysing identical samples with MA and HPLC, but was determined by comparing the values from several 
UK and USA datasets with the analytical results from 125 closely matched Dutch foods (3). A large variation in microbiological results from these datasets was reported and $27 \%$ was selected as the average conversion factor. Our results are in line with these findings, but also stress the large variability.

NEVO being the reference database for food composition data in the Netherlands is used for food consumption, for nutritional and dietetic counselling and by the food industry. High quality data and transparency in the data source are essential to provide fit for purpose data to users.

To comply with the international modus, we choose to change from HPLC to MA, although it can be argued that HPLC would still be a better method for folate analyses, as it can distinguish individual folate vitamers. In contrast, microbiological methods can only measure total folate and certain non-folate compounds could stimulate or inhibit bacterial growth and bias the results (3). New developments in techniques for folate analyses might yield improved procedures in the near future. However, before applying this to food composition databases, international consensus on the quality and comparability of such methods would need to be reached.

When comparing values from several databases major progress has been made since the research by Deharveng and Slimani $(5,6,7)$. The work by EuroFIR on standardising food description and value documentation allows for direct comparison of nutritional values in most European food composition databases (8). When comparing folate values, attention can be paid to the analytical method used as well as to the correct food identification and component descriptions. Component descriptions needs to be taken into account, whereas e.g. total folate can be the sum of food folate and folic acid or the factored summation that gives folate equivalents. Next to that users need to be aware of the large variability in both the folate content in food and in laboratory results.

Queries from several European food composition databases showed that the newly analysed Dutch MA values are within the range found in other databases. Before copying values, careful scrutinising is needed to see if values are fit for purpose, preferably using more detailed food and value description systems, such as LanguaL and EuroFIR thesauri $(21,8)$.

Food consumption research suggests that the folate intake of part of the Dutch population is too low (22). Updating folate values as performed in this project is likely to lead to higher habitual folate intake estimates for the Dutch population as a whole. In Dutch young children the median habitual intake increased by only $11-15 \%$. The conclusion drawn in the DNFCS for young children (16) remained unchanged with the new folate data. The median habitual intake of folate equivalents stayed below the adequate intake for 4-6-year-olds, allowing for no conclusion, as was explained earlier. For 2-3-year-old children the percentage having an inadequate intake remained low. On the other hand, there is the risk that microbiological folate values might overestimate folate intake (3) and hide inadequate intakes. This underpins the necessity of accurate and precise folate data. However, when comparing intakes the possible overestimation of folate intake will be similar in all countries using MA folate values in their food composition databases. As $28 \%$ of the analysed folate values in our database are still based on HPLC, finalising this update might result in a further increase of the estimated folate intake in the Netherlands.

\section{Conclusion}

The goal to update folate in the NEVO database to achieve comparable folate data within this database and among European databases has been achieved as far as possible with the present data. Comparability of folate data is an ongoing issue and work will continue when new values become available. Work done within EuroFIR, specifically with regard to detailed value documentation both for the Dutch and other datasets enables the (international) user to compare and judge on the usability and comparability of individual values and complete datasets.

The change from HPLC values to MA values for folate, in combination with new analytical MA values results in a somewhat higher estimated folate intake for young Dutch children. This effect is also expected for the general Dutch population.

Cooperation between chemical analysts and compilers of food composition databases and consensus on analytical methods that are fit for purpose needs to be an ongoing issue to produce high quality analytical values for food composition databases.

\section{Acknowledgements}

We thank Deborah Gibson-Smith for reviewing and correcting the manuscript.

\section{Conflict of interest and funding}

Funding for chemical analyses of 150 foods has been provided by the Dutch Ministry of Health, Welfare and Sports. Analytical data for approximately 30 types of bread has been made available for use in the NEVO database by Voorlichtingsbureau Brood (the Dutch Bread Information Centre). The authors declare no conflict of interest. 


\section{References}

1. Hoekstra J, Verkaik-Kloosterman J, Rompelberg C, van Kranen $\mathrm{H}$, Zeilmaker $\mathrm{M}$, Verhagen $\mathrm{H}$, et al. Integrated risk-benefit analyses: method development with folic acid as example. Food Chem Toxicol 2008; 46: 893-909.

2. Löwik MRH, Hulshof KFAM, Heijden LJM van der. Changes in the diet in the Netherlands: 1987-88 to 1992. Int J Food Sci Nutr 1998; 49: S1-S64.

3. Konings EJM, Roomans HHS, Dorant E, Goldbohm RA, Saris WHM, van den Brandt PA. Folate intake of the Dutch population according to newly established liquid chromatography data for foods. Am J Clin Nutr 2001; 73: 765-76.

4. Konings EJM. A validated LC method for the determination of folates in vegetables, milk powder, liver and flour. J AOAC Int 1999; 82: 119-27.

5. Deharveng G, Charrondiere UR, Slimani N, Southgate DAT, Riboli E. Comparison of nutrients in the food composition tables available in the nine European countries participating in EPIC. Eur J Clin Nutr 1999; 53: 60-79.

6. Slimani N, Charrondiere UR, van Staveren WA, Riboli E. Standardization of Food Composition Databases for the European Prospective Investigation into Cancer and Nutrition (EPIC): general theoretical concept. J Food Comp Anal 2000; 13: $567-84$

7. Slimani N, Deharveng G, Unwin I, Southgate DAT, Vignat J, Skeie G, et al. The EPIC nutrient database project (ENDB): a first attempt to standardize nutrient databases across the 10 European countries participating in the EPIC study. Eur J Clin Nutr 2007; 61: 1037-56.

8. EuroFIR. European Food Information Resource. http:// www.eurofir.net/home [cited 30 May 2010].

9. Statistics Netherlands (CBS) 2008. http://www.cbs.nl [cited 17 July 2008].

10. Hoofdbedrijfschap detailhandel (HBD) 2008. http://www.hbd.nl [cited 1 August 2008].

11. AOAC Official Method 2004.05. Total Folates in Cereals and Cereal Foods, Microbiological Assay-Trienzyme Procedure, First Action 2004. J AOAC Int 2005; 88: 5.

12. McCance and Widdowson's The Composition of Foods 2002 Integrated dataset (CoF IDS), Food Standards Agency, UK; 2006. http://www.food.gov.uk/science/dietarysurveys/dietsurveys/ [cited 6 July 2010].

13. NEVO table 2006, Stichting Netherlands Voedingsstoffenbestand, Voedingscentrum, Den Haag; 2006.
14. NEVO online version 2010/2.0, RIVM, Bilthoven, The Netherlands; 2010. http://www.rivm.nl/nevo_en/online/ [cited 6 July 2010].

15. Ocké MC, Hulshof KFAM, van Rossum CTM. The Dutch national food consumption survey - 2003. Methodological issues. Arch Public Health 2005; 63: 227-41.

16. Ocké MC, van Rossum CTM, Fransen HP, Buurma-Rethans EJM, de Boer EJ, Brants HAM, et al. Dutch National Food Consumption Survey-Young Children 2005/2006. RIVM-report 350070001. Bilthoven, the Netherlands: RIVM; 2008. http:// www.rivm.nl/bibliotheek/rapporten/350070001.html [cited 6 July 2010].

17. Nusser SM, Carriquiry AL, Dodd KW, Fuller WA. A semiparametric transformation approach to estimating usual daily intake distributions. J Am Stat Assoc 1996; 91: 1440-9.

18. Institute of Medicine, Dietary reference intakes. Applications in dietary assessment. Washington DC: National Academy Press; 2000

19. Koontz JL, Philips KM, Wunderlich KM, Exler J, Holden JM, Gebhardt SE, et al. Comparison of total folate concentrations in foods determined by microbiological assay at several experienced U.S. commercial laboratories. J AOAC Int 2005; 88: 805-13.

20. Puwastiena $P$, Pinprapaia N, Judprasonga K, Tamurab $T$. International inter-laboratory analyses of food folate. J Food Comp Anal 2005; 18: 387-97.

21. LanguaL, The international framework for food description. http://www.langual.org/ [cited 6 July 2010].

22. Health Council of the Netherlands. Towards an optimal use of folic acid. Publication no. 2008/02 The Hague: Health Council of the Netherlands; 2008 .

23. Vahteristo LT, Lehikoinen K, Ollilainen V, Varo P. Application of an HPLC assay for the determination of folate derivates in some vegetables, fruits and berries consumed in Finland. Food Chem 1997; 59: 589-97.

\section{*Susanne Westenbrink}

RIVM

P.O. Box I

Bilthoven

3720 BA

The Netherlands

Email: susanne.westenbrink@rivm.nl 